\title{
Uncertainty as the Substratum of Entrepreneurship
}

\author{
Alexey B. Kurlov \\ Ufa State Aviation Technical University \\ Askar G. Khairullin \\ Kazan Federal University, Branch in Naberechnye Chelny \\ Email: askar58@mail.ru
}

Doi:10.5901/mjss.2015.v6n3s5p71

\begin{abstract}
The article addressed the stochastic issues of modern business environment, the content of the concepts of uncertainty and risk. The basis of the concept of business initiatives are defined through the prism of ontology of venture activities. It is proved that the risk enhances the capabilities of a subject and increases its adaptive capacity due to the timely actions. That is why the risk becomes the property of a particular resource that can improve the sustainability of a business entity acting in the face of uncertainty. The sources of uncertainty and risk are shown, being conditional to the implementation of particular subject activity in the business sector. From the standpoint of this study, risk is considered as an attribute of entrepreneurship, which is sure to arise in situations where alternatives are possible, realized in many outcomes. There is uncertainty about a particular outcome, and at least one of the outcomes is harmful. In a broad sense, the risk is understood as a solution associated with the choice of a wide variety of complementary or alternative options, the result of which is internally and unavoidably inherent with uncertainty. Thus, the risk is a characteristic of solutions made by the subject, reflecting the uncertainty of achieving the objectives of its activities. Uncertainty, acting as a substrate of entrepreneurship, is implemented in various alternative ways of achieving subjective goals. Under these conditions, the venture of business activity is manifested most clearly, and the risk is acquiring attribute qualities as an immanent feature of the modern business environment.
\end{abstract}

Keywords: social interaction, social dynamics, uncertainty, entrepreneurship, entrepreneurial risk, stochastic attributes

\section{Introduction}

Current article draws on the standpoint of one of the sections of the monograph written by Alexey B. Kurlov (see: Kurlov, 2007), which are being represented here in the context of the renewed intention. Professor Askar G. Khayrullin have worked on the development of certain aspects of the concept being presented, resolving some organizational issues, adopting the material to a new audience. Actualization of the problem boils down to the fact that today the development process of social existence is characterized by a pronounced dynamics of social change, the laws which are not always possible to explain by using the principles of traditional deterministic methodology. It is for these reasons that we make the transition from the use of the principle of development as a means of knowing the object to the transformation process of the development of business activities taking place under conditions of uncertainty in to the actual subject of social studies.

Considering the features of the entrepreneurial activities of the various socioeconomic objects, we emphasize that the phenomenon of stochastic dynamic property has an attribute of complex open systems, actively interacting with the external environment. This phenomenon manifests itself in all stages of development of the object, starting from the inception phase of multiple choice alternatives, with the expressed uncertainty in the trajectory of development, which is a desire to streamline the hierarchical structure and remove the uncertainty in the form of homeostatic balance, concluding with its bifurcation failure to disequilibrium to a new freedom of choice among the many evolutionary (or even revolutionary) development scenarios. Phenomena fluctuations, bifurcations, dissipation, followed by reduction of the stability of the system, the formation of its hierarchical structure and motion to homeostasis with possible disruption to the disequilibrium - all of the above are periods of the self-organization cycle of business activities, which can manifest itself only in terms of openness and the relative uncertainty of socioeconomic system. 


\section{Traditional Paradigm of Socioeconomic Systems}

The traditional paradigm of socioeconomic systems represented in numerous works of philosophers, sociologists and economists is nowadays in contradiction with the objective tendencies of development of the real economy, both at micro and macro levels. Today's reality is that every stakeholder is experiencing an increasing aggression of the environment in the form of uncertainty - the fundamental impossibility of clearly and unambiguously prolong any action on any term. Stochasticity as an attributive property of socioeconomic objects not being as vivid previously, manifests itself as a source of this aggression. The subject of entrepreneurial activity is forced to operate in conditions of objective uncertainty generated by the ever-increasing complexity of systems and complexity of socioeconomic intersubjective organizational and economic relations. It is obvious that efficiency of business in this situation has a significant variation, and the process of making any decisions is fraught with risk. At the risk of unexpected costs and even the risk of losing their status, which they risk by trying to bring a higher level of quality.

The main legitimization prerequisites of a new development paradigm of the business environment, adequate to modern realities, are the following general methodological provisions:

- Developing socioeconomic object is un-identical to itself, is the subject to constant modification, while the unique "development ladder" of each object introduces additional uncertainty into the overall structure of socioeconomic relations;

- The relation of states and events mediated by the process of "transition" in which there are no strict laws, the situation of choice is not predetermined, which eliminates the possibility of logical deducibility of subsequent states based on the prior state;

- Each object is developing on the basis of several types of interactions, which makes a high degree of randomness;

- The risk is an attribute of entrepreneurial activity and is defined by the need to eliminate uncertainty in the resolution of a given situation in the field of business.

Orthodox paradigm of economic risks was based on the principles of neutralizing - leaving them aside. However, it is almost impossible to get away from risk, carrying out activities in the face of uncertainty. Therefore, an entity must engage in an endless stream of objective uncertainty, learn to operate in within, turn this objective reality in the material of productive construction business technology by acknowledging the risk. This idea is put in the foundation of a new paradigm of understanding the essence of entrepreneurship, as well as methodological research built on this basis. The overall composition of this epistemological system is as follows:

- Proves that stochasticity is an attributive sign of modern socioeconomic environment;

- A product of this environment is the uncertainty, redundancy, high variability of combinations of evolutionary development of an object of entrepreneurship;

- As part of this space a subjective activity is realized, which is due and, at the same time, burdened by the knowledge that falls beyond an absolute reality;

- The actual combination of the two forms of redundancy (uncertainty) is a substrate of venture entrepreneurship;

- The acknowledged risk is becoming a major element of entrepreneurship and, simultaneously, a prerequisite for its conflict-free existence in a stochastic environment. The risk, due to its urgent action, extends the capabilities of a subject and increases its adaptive capacity. That is why the risk becomes the property of a particular resource that can improve the sustainability of a business entity acting in the face of uncertainty.

It must be emphasized that overcoming uncertainty (by means of venture activities) by no means assumes a complete removal of the latter. It would be fraught with some loss of freedom. Subject always seeks to establish only an optimal balance between certainty and uncertainty in the normal course of business. Certainty characterizes the potency of the subject in achieving goals, while uncertainty characterizes the range of possibilities within which results may deviate from the standard target.

Describing the ratio of certainty and uncertainty in the real practice, it is noted that one aspect of this relationship is a situation of choice. If a person has a multiplicity of choices, then there is always uncertainty in selecting the correct approach. Uncertainty increases with the number of elements in the set. In a situation of an unknown number of elements, the degree of uncertainty using probability opportunities is used for measurements (Gott, 2001).

As for the working definitions of certainty and uncertainty categories, we can highlight the following: uncertainty the category marked by the transformation of the manifold possibilities into reality; the presence of communication, interaction between the properties and conditions of phenomena, the absence of sharp edges between them. At the 
same time certainty defines the relative independence of the properties and states of objects through the manifestation of the need for unambiguous transitions of possibilities into reality. It is also important to note that these interrelated concepts do not rise before each other, but are set in coordination (Ursul, 1999).

\section{Ontology of Uncertainty and Risk in Entrepreneurship}

Entrepreneurship is implemented in an environment of ambiguity of real socio-economic processes, a variety of methods and options for the conversion of opportunities into reality, the variety of possible conditions and situations for implementing this solutions, which may be the future of an economic entity. At the very moment of decision-making, it is impossible to obtain a complete and accurate knowledge of the remote environment in time to implement the decision, on all existing or potential internal and external factors. All this is an expression of uncertainty as an objective form of existence of economic activity. Objectively, there are fundamentally unavoidable uncertainties existing in decisionmaking, leading to the fact that the risk never equals zero. The consequence of this is the uncertainty in the reachability of the goal, and as a result, the intended target is not achieved.

There are several sources of uncertainty, but above all - it is incompleteness, inadequacy of our knowledge about the economic sphere and the world around us. This kind of uncertainty follows a man for a long period of time, starting with the very first decisions. Ignorance of the laws of nature has impact on the production activity, affecting the efficiency of the economy. Another source of uncertainty is a randomness (or coincidence), different results under similar conditions that cannot be predicted in advance. It is impossible to plan any given situation. Equipment failure and sudden changes in demand for products, the sudden failure of supply of raw materials - all is a manifestation of randomness. There is a third reason for uncertainty - resistance, which may manifest itself in the case of breaching the contractual obligations by suppliers under the influence of uncertainty in demand for products, difficulties in its marketing.

One of the first scientists to draw attention to the problem of uncertainty in the framework of modern economic theory was an American economist Frank H. Knight (1921). In his article, "The concept of risk and uncertainty", published in 1921, he noted that there are two types of probability: mathematical and statistical. The probability of the first type is determined by the common, predefined principles. "The mathematical probability is absolutely homogeneous classification of cases, identical in all" (Knight, 1921, p. 21). The probability of the second type can only be determined empirically. The statistical probability of an empirical assessment of the frequency display relationship between statements not being decomposable in the volatile combination of equal alternatives. The first type of probably is very rare in the business, and the second is typical for the business sector. The first type is amenable to an unambiguous measurement to measure the two required subjective assessment.

It is very difficult to come up with an example of 'risk' in business, in relation to which one could calculate in advance the distribution of different possible outcomes. To solve the problem (if the decision is possible at all) it is required to study the empirical results. It is obvious that many of the risks can be brought to a large extent of predictability by the statistical groupings, while the other equally important part cannot follow this procedure. Opinions that are responsible for our actions in everyday life and manage the solutions of the responsible managers in business, for the most part have little in common with the conclusions reached by the comprehensive analysis and accurate measurement. Thought processes in these two classes of cases are quite different. In everyday life, they are mostly unconscious.

Verification of opinions and estimates by the errors must be distinguished from both types of probability, because they reflect no possible way of whatsoever sufficiently homogeneous groups of cases to be formed, the analysis of which would allow quantifying the value of the true probability. For example, business decisions are, in general, the unique situations, so that they cannot be subjected to statistical grouping to determine the approximate probability of a particular outcome. The concept of objectively measurable probability is simply inapplicable. The confusion arises from the fact that, in practice, we assess the validity and reliability of our opinions, and such an assessment in its form is similar to the probabilistic judgment. It is this uncertainty that gives true economic organization a characteristic form of 'enterprise' and explains the existence of a specific income of an entrepreneur.

To preserve the distinction between measurable and unmeasurable uncertainty, which we discussed above, the term 'risk' will be used to refer to the first type of uncertainty and the term 'uncertainty' - for the second. The word 'risk' is generally very loosely applied to any type of uncertainty discussed in terms of possible adverse outcome, and the term 'uncertainty' - in relation to the possibility of a favorable outcome; we speak of the 'risk' of losses and 'uncertainty' of winning (i.e. gains). The practical difference between the categories of 'risk' and 'uncertainty' is that in the first is the distribution of results in the group is known (which is achieved by means of a priori calculations or statistical study of previous experience), and the second is not, and it is most often caused by the inability to carry out grouping of cases, as might be considered the unique situation. 
It should be noted that when considering the individual case there is no difference between the measurable and immeasurable risk of uncertainty in terms of behavioral decision making. The individual gives an assessment of the accuracy of probabilistic judgments form 'a success in cases of b' (which corresponds to an equation of a/b), and a subjective attitude to this assessment, similar to any other type of probability (Vladimirov et al., 2000).

Uncertainty as a socio-economic definition as a sign of modern socio-dynamics still generally seemed as something homogeneous, which has no structural elements. Such a situation can be taken as one of the many paradoxes of economic theory, which, like any other science, often builds the upper floors of its knowledge base, not fully finished laying the foundation. In this case, the situation requires special attention. Uncertainty remains in the theory of uncertainty - this is a very specific scientific paradox. Of course, it is not just the obvious terminological reminiscences. There are far more substantial grounds for concern.

Can we talk about scientific and practical value, validity and reliability of the theory of uncertainty and risk if their very nature, origin, and value is unclear? Can heterogeneous and diverse risks arise from a homogeneous and uniform uncertainty? What is the source, the cause, time and how does the uncertainty arise? Can the uncertainty be considered (as it is done today) as a homogeneous, unchanging, and fixed irrespective of the place and time of its occurrence? When and why as additional information and new knowledge acquisition happens, uncertainty does not decrease, but rather increases? The answers to these and many other questions depend on its ability to show whether that uncertainty has its own genetic, structural and functional definitions and models.

Tasks of disclosure of the nature of uncertainty are of particular relevance to entrepreneurship. But the development of these issues is carried out mainly in applied aspects, in particular in relation with the use of economic and financial information technology. On general methodological level, their development is limited to the interests of the foundations of the theory of information, and management. Socio-philosophical level, these problems are not yet well developed.

Perceptions of uncertainty and risk are abstractions based on the study, description and explanation of many empirical observations. The question is how the researcher receives meaningful interpretation from this empirical experience, which leads eventually to an independent scientific concept. It is even more difficult to answer the question of how to use the already known concepts of uncertainty and risk, to come to a qualitatively different, new finding that are consistent with the results of new observations of researchers. The answers to these questions depend on the accepted, rejected or transformed perceptions of the uncertainty and risk. Summing up the traditional view, one might select a number of provisions that were formed in the process of understanding the dynamic nature of the various manifestations of socioeconomic activities.

From the standpoint of current research, risk is an attribute of entrepreneurship that is sure to arise in situations where alternatives are possible, realized in many outcomes. There is uncertainty about a particular outcome, and at least one of the outcomes is unfavorable. The risk is understood as a decision associated with the choice of a wide variety of sufficient complementary or alternative options. The result of this decision internally and unavoidably inherent to uncertainty. Thus, the risk is a characteristic of decisions of the subject, reflecting the uncertainty of achieving the objectives of its activities.

At present, there is no clear understanding of the nature of risk. This is due, in particular, to the multidimensionality of this phenomenon. Risk is a complex phenomenon, having a plurality of distinct and sometimes opposite real basics. This leads to the possibility of different interpretations of this phenomenon: a) potential numerically measurable possibility of losing. The concept of risk is characterized by the uncertainty associated with the possibility of the implementation of activities in adverse situations and consequences; b) losses, damages, shortfalls of the projected revenues, profits, and uncertainty of the financial results in the future; c) self-expression of the probability of events leading to losses; d) the probability of loss of valuables (financial, commodity resources) as a result of the activities, if the situation and conditions of the activities will change in a direction different from that of the estimates.

So, the risk is a phenomenon that has a heterogeneous (objective - subjective) nature. This implies that the sources of risk, their causes, as well as the main factors that influence not only the level but also at the "habitat" of risks, which is the uncertainty of the objective conditions, states and processes on the one hand, and uncertainty of subjective goals, attitudes, behavior of the subjects of business activities on the other.

Thus, the nonlinear nature of development of the socioeconomic systems, within which there is a subjective business activity, creates uncertainty. It manifests itself in a variety of alternative ways of achieving subjective goals. This multiplicity is a clear sign of a complex socio-economic object, which genetically carries a redundant choices in the trajectory of further development. Moreover, the effect of the subjective factor, in the form of excess knowledge, adds additional uncertainty to the development of a situation. Obviously, these circumstances cause the phenomenon risk activity that is a natural consequence of being subjective in terms of relative uncertainty. 
Uncertainty is generally understood as something unspecified, not entirely clear, unrestricted, vague, completely or partly unknown, unpredictable changing, unstable, indefinable, inexplicable, strange, etc. In other words, the uncertainty is all that does not or cannot have all the necessary and sufficient definitions, characteristics, descriptions, justifications, explanations, predictions of future states and changes. Consequently, the uncertainty is, first of all, the lack of objective information, in terms of which the entrepreneur is forced to act.

It is known that the development of man and nature, society and the economy happened and is happening due to the huge number of random events. Accident (randomnicity) is the only way to implement changes in the conditions of uncertainty, with a diversity and redundancy, diversity of redundancy and redundancy of diversity all at the same time. Of course, the social (socio-economic) reality is always richer than any of the most complete its descriptions, so it is necessary to recognize the existence of chance (randomnicity) in its bosom, which is due to redundancy, at the level of objective existence, and at the level of consciousness of the subject of socio-economic change.

While uncertainty is a virtual array of opportunities and development sources, the accident is the primary means to implement these features. Thus, the need arises for a particular situation, the implementation of formal, abstract and real opportunities. Therefore, if individual entrepreneur can affect the relationship between the spheres of determination and chance, it is done by the use of uncertainty as a kind of substrate of socio-economic activity.

External environment of the organization is the most often states as the source of uncertainty. That is, this source is seen as external to the subject of business activities, the decision maker. While the external environment has various definitions. Traditionally scholars distinguish two levels of the environment: the micro-level - the target environment of an object (other business actors that directly affect the goals being set and achieved) and macro-level (all external influence, including political, legal, religious, etc. that determine the conditions of operation and development of socioeconomic system).

Factors contributing to the uncertainty of the environment often referred to as:

- Lack of information about the external effect, which leads to certain difficulties in the intra-decision-making process;

- The inability to determine how likely an external influence will affect the success or failure of decision-making units;

- Lack of information about the consequences of a wrong decision or action.

It is obvious that the parameter of 'certainty-uncertainty' is characterized not so much by the environment itself, but by the degree of completeness of the information held by the decision maker. Hence, the ability of the organization to successfully adapt to their environment to the greatest extent depends on the ability of the decision-makers to predict the future state of the environment. Thus, the mechanism for the elimination of uncertainty appears to be non-standard solution based on the intuition of managers and leading to a rational adaptation of an organization to its microenvironment (Popova, 2006).

Considering the phenomenon of uncertainty from the perspective of macro-business activities, most analyzes end up in neoinstitutional theory and the theory of organizational ecology. The first theoretical field (e.g. see J. Meyer, B. Rouan, P. Di Maggio, V.R. Scott, U. Pauel) deals with macro environment as a source of uncertainty. At the same time, it is the macro-environment that sets the conditions for the activity that determines its maximum perspectives and the result (the concept of institutional isomorphism). On the other hand, the survival of the organization requires a certain level of performance. Thus, the functioning and the change in the socioeconomic system is the result of actions that enable the balance between satisfying the requirements of adaptation to the regulatory standards being used and requirements of organizational and technical efficiency. This is possible if the organization is a complex structure consisting of sectors that perform different tasks. However, a problem arises in the linkages between these tasks and, therefore, integration of organizational structure of entrepreneurship. This is a major source of uncertainty in the business activities of the organization. Just so, the environment produces uncertainty and directly changes external conditions of an organization, and indirectly, by creating the problem of linkages to adapt to the changing requirements of external conditions within the organization.

To ease the impact of uncertainty, it is proposed to apply the strategy of 'free communication': organizations that adapt their structures to the institutional environment reduce internal coordination and control in order to maintain their legitimacy. Therefore, the organization receives a chance to respond to conflicting demands coming from its environment. In this case the adaptation to external aggression only symbolically, formally, through the creation of sub-structures that resolve (or, at least, demonstrate this possibility) the problem, namely - to adapt to the changes of the macro environment. The activity of these peripheral structures remains 'loosely coupled' with the key activities of the organization, allowing it to maintain a modicum of efficiency in order to stay in business.

In addition, the macro-level research enables to structure the notion of 'efficiency' and not to reduce it in its entirety 
up to the technical efficiency (achieving specific goals with minimal cost). Sustaining the committed of a business organization to rationality characterizes the structure as high-level, which can maximize the long-term efficacy. Commitment to the greater institutional goals, rather than rationality on microscopic daily activities, enables the effective achievement of social goals. It is this ability of business organizations, as a long-term target groups, is their advantage over short-term types, specially designed for a specific purpose, which tend to have greater technical efficiency and no tendency to become the aim for themselves. A similar view is presented in the theory of organizational ecology (see M. Hennen, J. Freeman).

These researchers emphasize the organizational interaction with the macro-environment and see external environment as a source of uncertainty. However, this concept takes into account the factor of chance (not absolutizing it). In addition, organizational environmentalists believe that the symbolic adaptation, creation of peripheral structures and weakening of the links between them and the key activities of the organization are possible only in the case of resource abundance. In the case of reducing the resource base, the concept of institutional isomorphism focuses on the identity of the target functions of the various business entities, which cannot adequately describe the socio-dynamics of development of organizational forms needed for a successful business. This causes the selection of the unit of analysis in the framework of this approach: analyzes account for a "population" of organizations, rather than a separate organization. In essence, the similar types of organizations within a limited system. Moreover, this view point does not exclude the traditional demands made by different versions of the theory of rational choice - to analyze the actions of the individual decision maker. The combination of these positions is reflected in the three-dimensional evolutionary model (Goodman \& Kurke,1982).

The first stage are the changes that occur in individual organizations in response to changes in the external environment. However, there is no reason to believe that these changes are the result of rational choice. After the thesis of R. Merton on ambiguity and multiplicity of the consequences of any social action and the concept of 'bounded rationality' developed by Herbert $\mathrm{A}$. Simon, the recognition of restrictions on rational choice theory became took a central place in organizational theory. Organizational ecologists believe that the changes taking place in each organization might be the result of chance, or imitation, or successful solution of a manager, but from an evolutionary point of view, it does not matter. Thus, the randomness factor is considered at the level of a single subject.

The second stage is the selection of best options for changes at the level of 'population' of organizations and the completion of the general fund of effective standard actions on their due. This fund contains only the steps that proved to be the most successful. They lead to a balance requirements of institutional isomorphism and technical efficiency and enable the organization to strengthen its existence in the social environment and in current configuration of microenvironment in a given situation.

The third stage is memorization of the best options and expansion of behavioral repertoires of each individual organization in the population. Reduction of the factor of chance and the opportunity to resolve the problem of uncertainty for each individual organization in the population depends on the manner in which, the richness of its behavioral repertoire in different situations will help it overcome the aggression of the environment.

\section{Concluding Remarks}

We justify the fact that it is impossible to avoid risk averse while carrying out activities in the face of uncertainty. Therefore, it is necessary to engage in an endless stream of objective uncertainty, to learn to operate within this uncertainty, making this objective reality in the material of productive design of business technology. Thanks to these urgent actions, the risk extends the capabilities of a subject and increases its adaptive capacity. That is why the risk becomes the property of a particular resource that can improve the sustainability of a business entity acting in the face of uncertainty.

To conclude, we emphasize once again that subjective entrepreneurial activities within the nonlinear nature of the development of economic systems create uncertainty. Being a substrate of entrepreneurship, it is implemented in a variety of alternative ways of achieving subjective goals. Under these conditions, the venture capital of entrepreneurship is most clearly manifested, and the risk acquires the attribute qualities as an immanent feature of the modern business environment.

\section{References}

Goodman, P.S., \& Kurke, L.B. (1982). Studies of change in organizations: A status report. In P.S. Goodman and Associates (eds.). Change in organizations: new perspectives on theory, research and practice. (pp. 22-24). San Francisco, CA: Jossey Bass. 
Gott, V.S., \& Ursul, A. D. (1971). Definiteness and uncertainty of a nature and scientific knowledge. Moscow: Nauka. [in Russian] Hebert, R., \& Link, A.N. (1988). The Entrepreneur: Mainstream views and radical critiques. New York: Praeger.

Knight, F. H. (1921). Risk, uncertainty and profit. Boston: Houghton Miffin Co.

Kurlo, A.B., \& Petrov, V.K. (2014). Methodology's of information analytics. Moscow: Prospectus. [in Russian]

Kurlov, A.B. (2007). Philosophy of Business. Ufa: Kitap. [in Russian]

Mills, C.W. (1956). The American Middle Classes. New York: Oxford Galaxy Book.

Popova, E.P. (2006). Problem of uncertainty of structural changes in the organizations. Personality. Culture. Society, 1, 41-59. [in Russian]

Ursul, A.D. (1999). Information. Methodological aspects. Moscow: Nauka. [in Russian]

Vladimirov, V.A., Vorobiev, U.L., \& Salov, S.S. (2000). Risk Management: Risk. Sustainable development. Synergetics. Moscow: Nauka. [in Russian]

William, J. (1975). Pragmatism. Cambridge: Harvard University Press. 
